\title{
Study on Bias Control of Memristor Multistablity System
}

\author{
Hongyan Zang, Lili Huang, Yanling Wang, Tengfei Lei * \\ Collaborative Innovation Center of Memristive Computing Application (CICMCA), Qilu Institute of Technology, Jinan 250200, China
}

\begin{abstract}
In this paper, we study a memristor chaotic system with bias control. Based on the analysis of basic dynamic behavior, the hidden attractor coexistence and multi-stability of the non-equilibrium memristor chaotic system are verified, the bias control based on DC power control is studied, and the polarity control of the output variable is realized by using the change of parameters. The sinusoidal function is introduced as the bias periodic function, so that the system can realize signal polarity control only by changing the initial conditions. The analog circuit of memristor chaotic system is designed and verified by simulation..
\end{abstract}

\section{Introduction}

In 1971, Chua proposed a memristor to represent the relationship between charge and flux from the view of symmetry when analyzing the relation among the voltage, current, charge and flux. Memristor, as a new electronic element with memory effect, has been widely applied in many fields and has become a research hot focus. In 2008, HP laboratory released a $\mathrm{TiO} 2$ charged memristor [1] declaring that a memristor was found. A large number of researches on memristor models and typical chaotic systems have been published, making memristor widely studied in storage, computing, neural network, artificial intelligence and other aspects. Memristors are introduced as feedback terms into Chae circuits, chaotic systems, or directly replace resistors in chaotic systems to construct memristors chaotic systems. The hidden attractors[2], coexisting attractors, multistability[3], symmetry[4], amplitude modulation[5], and offset boosting studied in chaotic systems also become the research problems of amnesic chaotic systems. It was found in literature [6] that multiple attractors also coexist in neural networks.

The hidden attractor is a hot research point at present, adding memristor in chaotic system will make the system have an equilibrium set on some coordinate axis, so it is necessary to study the hidden attractor in chaotic system. In [7], it is the case that all equilibrium points fall on a plane hyperbola of infinite multiple equilibria. In [8], the hidden attraction subsystem with complex transient behavior is studied for the chaotic system with line equilibrium point [9], the hidden attraction subsystem without equilibrium point is studied. The existence of hidden attractors may lead to sudden large chaotic vibrations, which may have disastrous consequences [7], which is also an important reason for the study of hidden attractors.
Without changing the oscillation characteristics, the output polarity of the signal can be adjusted by adding a DC voltage source [10]. In this paper, we study a bias control system of memristor chaos, and verify the coexistence and multistability of hidden attractors by analyzing the basic dynamics behavior. By introducing the sine function as the bias periodic function, the signal polarity control can be realized only by changing the initial conditions.

\section{Model of memristor chaotic systems}

\subsection{Formatting the title Mathematical model}

On the basis of the third-order system, the memristor function is taken as the feedback to construct the fourthorder memristor chaotic system. Its mathematical model is expressed as:

$$
\left\{\begin{array}{l}
\dot{x}=-b z \\
\dot{y}=z^{2}-a \\
\dot{z}=\operatorname{exW}(\varphi)-y z \\
\dot{\varphi}=x-\varphi
\end{array}\right.
$$

In the formula, $x, y, z$ and $\varphi$ are four state variables. $a$, $b, e$ are three system parameters, $\mathrm{W}(\varphi)$ refers to generalized magnetron memristor, and the memristor model can be described as:

$$
\left\{\begin{array}{l}
\mathrm{i}=\left(\mathrm{m}+\mathrm{n} \varphi^{2}\right) \mathrm{u} \\
\dot{\varphi}=\mathrm{u}-\varphi
\end{array}\right.
$$

Where $m$ and $n$ are internal parameters of the memristor, which are positive. 


\subsection{Basic dynamic analysis}

Analysis the dissipation of system (1), $\nabla \mathrm{V}=\frac{\partial \dot{\mathrm{x}}}{\partial \mathrm{x}}+\frac{\partial \dot{y}}{\partial \mathrm{y}}+$ $\frac{\partial \dot{z}}{\partial z}+\frac{\partial \dot{\varphi}}{\partial \varphi}=-1<0$. Indicates that the system is dissipative. Let, $\dot{x}=\dot{y}=\dot{z}=\dot{\varphi}=0$, the system does not have any mathematical solution, that is, the proposed memristor chaotic system has no equilibrium point, and the system has hidden attractors.
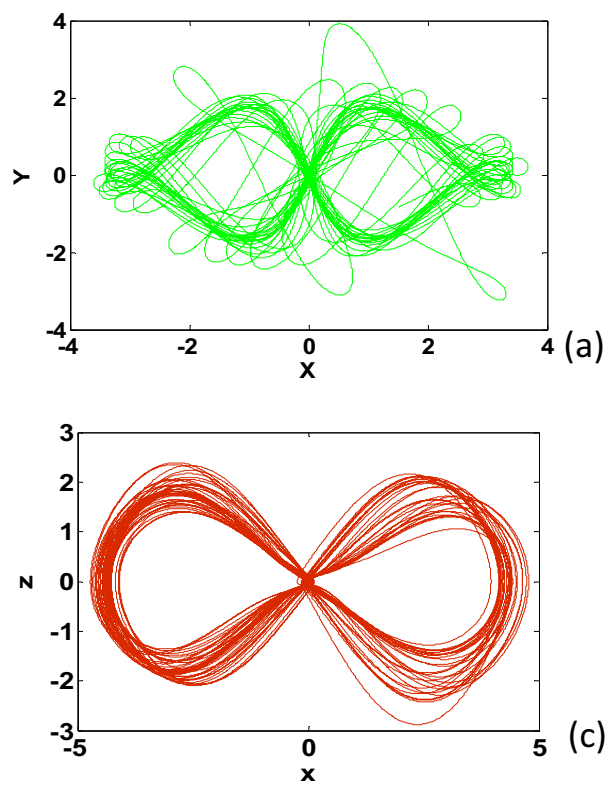

(c)

Figure 1. Phase trajectories of memristive system (1) when IC $=(-1,-2,0,0)$ (a) $x-y$ plane, (b) $y-z$ plane, (c) $x-z$ plane and (d) $y-u$ plane.

\section{Multistability analysis}

When parameter $b$ varies from 0 to 2 and other parameters keep unchanged under the initial condition (-

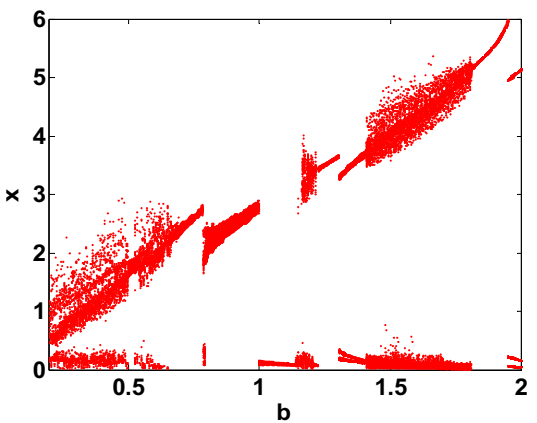

(a)

Figure 2. Bifurcation diagram and Lyapunov exponents when $a=0.5, e=1, m=0.5, n=0.01$, and $b \in$ $[0,2]$. (a) Bifurcation diagram. (b) Lyapunov exponents.

Multistability indicates that a system with fixed parameters and different initial conditions can give different attractors. In system (1), when $a=0.8$, periodic states and different chaotic states exist in the four different initial states. However, the phase trajectories are basically same each other in the case of $\mathrm{IC}=\left(\begin{array}{lll}0 & 1-2 & 0\end{array}\right)$
When parameters $a=0.8, b=1, e=1, m=0.5, n=0.01$, the Lyapunov exponents of system (1) can be calculated by Wolf[12] algorithm as follows: LE1 $=0.6655, \mathrm{LE} 2=$ 0.0045495 , LE3 $=-0.47521$, LE4 $=-1.1491$, the system has chaotic attractor. Matlab is used for numerical simulation, and the phase trajectories of each plane are shown in figure 1.
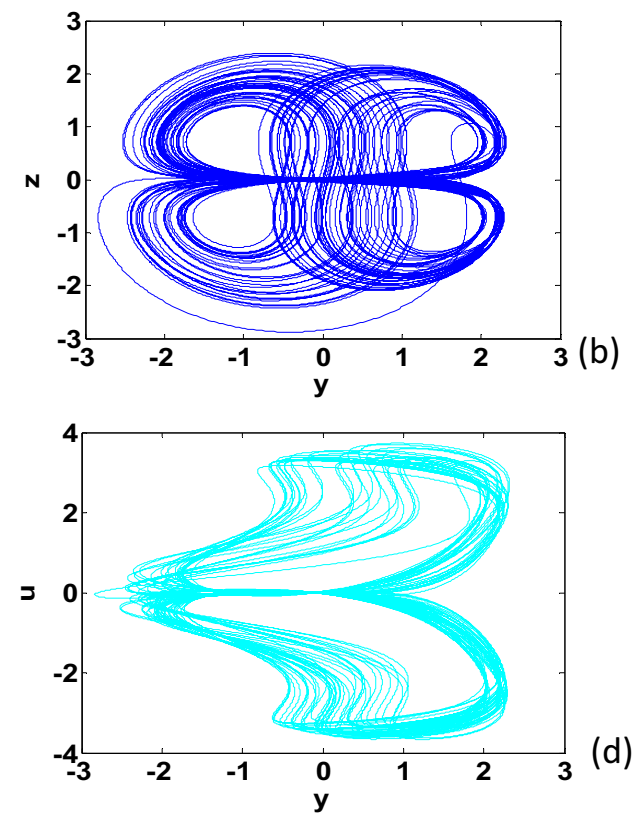

$1,-0.1,0,-0.7)$, the bifurcation diagram of the state variable $\mathrm{x}$ and the corresponding Lyapunov exponent spectrum are shown in figure 2 .

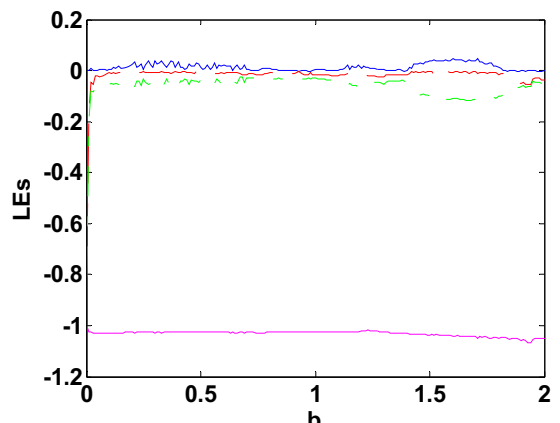

(b) and (0 0-2 7), when $a=0.1$ or $a=0.5$. Among them, when $a=0.1$, chaotic states and periodic states coexist, and when $a=0.5$, three different chaotic states coexist. The coexistence phenomenon of attractors in system (1) in numerical simulation is shown in figure 3. 


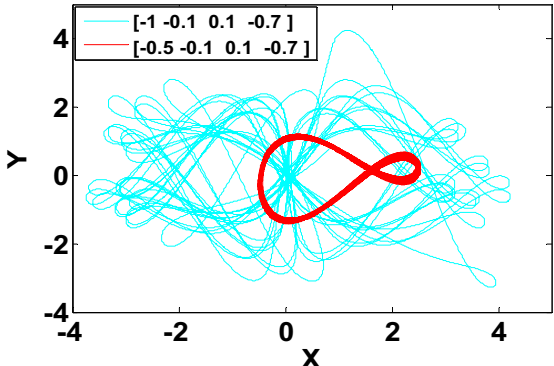

(a)
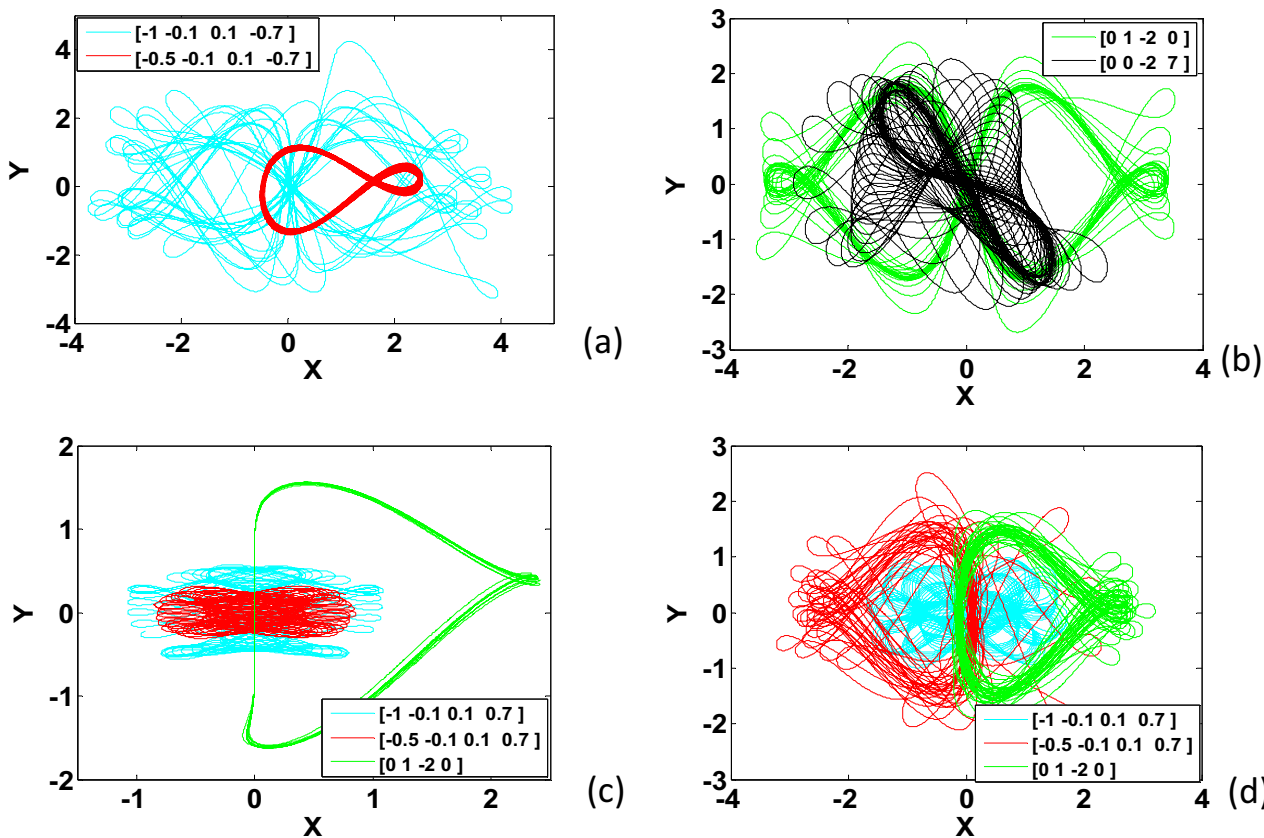

(c)

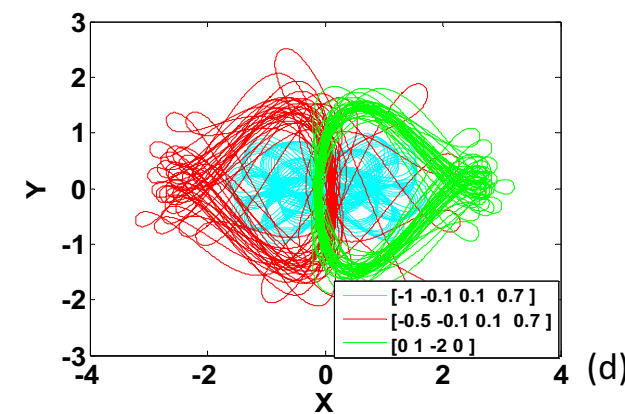

Figure 3. The coexistence of multiple attractors in system (1), where $b=1, e=1, m=0.5, n=0.01$, (a) $a=0.8$, the trajectories is blue, when IC $=(-1-0.10-0.7)$, the trajectories is red, when $\mathrm{IC}=(-0.5-0.1$ $0-0.7)$, (b) $a=0.8$, the trajectories is green, when $\mathrm{IC}=\left(\begin{array}{lll}0 & 1-2 & 0\end{array}\right)$, and the trajectories is black, when $\mathrm{IC}=\left(\begin{array}{ll}0 & 0-2\end{array}\right)$, (c) $a=0.1$ (d) $a=0.5$.

\section{Bias control}

\subsection{Bias control based on voltage source}

The change of parameters is used to control the polarity of y variable in the system, that is, to adjust the output polarity of the signal by adding a DC voltage source. System (3) is generated by offseting of the variable $y$ in system (1).

$$
\left\{\begin{array}{l}
\dot{x}=-b z \\
\dot{y}=z^{2}-a \\
\dot{z}=x W(\varphi)-(y-d) z \\
\dot{\varphi}=x-\varphi
\end{array}\right.
$$

The attractor phase trajectory of the system under the change of bias parameter $\mathrm{d}$ and corresponding initial value is shown in figure 4 . From left to right, it is $d=-10$, 0 , and 10 .The initial values are $(2,-10,5,1)$ and $(2,0,5$, $1)$ and $(2,10,5,1)$ respectively. The polarity of signal $y$ can be controlled by the change of parameter $d$ and initial conditions, and the attractors corresponding to the bipolarity, negative polarity and positive polarity can be selected from the blue-green, blue and red attractors.
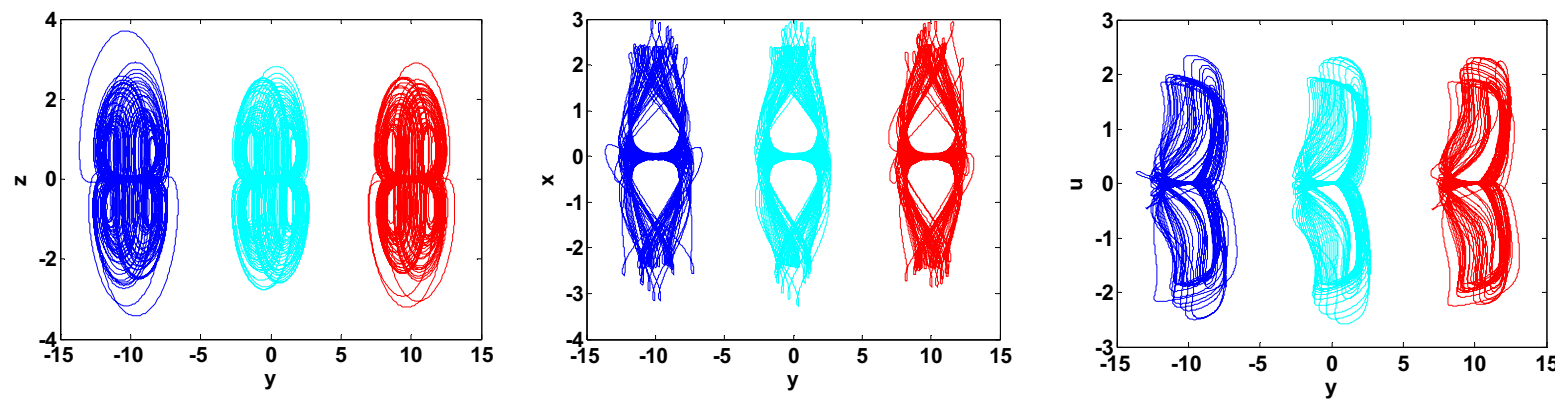

Figure 4. Attractors based on voltage source bias of system (3).

\subsection{Bias control based on initial conditions}

In order to make the system realize signal polarity control only by changing the initial conditions, the sine function is introduced as a bias periodic function, and the system (4) can be obtained as follows:

$$
\left\{\begin{array}{l}
\dot{x}=-b z \\
\dot{y}=z^{2}-a \\
\dot{z}=x W(\varphi)-h \sin (\mathrm{g} y) z \\
\dot{\varphi}=x-\varphi
\end{array}\right.
$$


When the parameters $a=0.5, b=1, h=5$, and $g=1$, seven attractors obtained are shown in figure 5 when $k$ is $0,1,2$. Therefore, introducing the sine function can

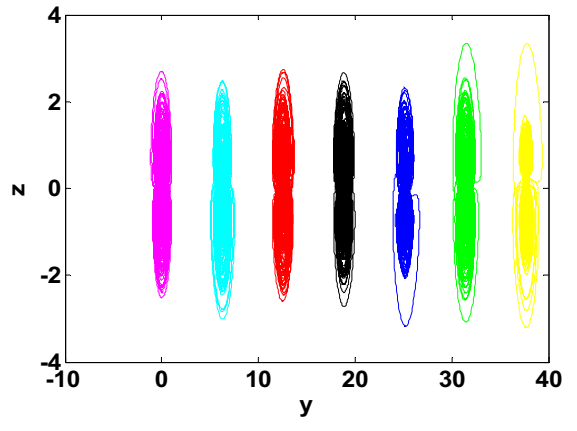

change the output polarity of the state variable $y$, only by changing the initial value of the state variable $y$.

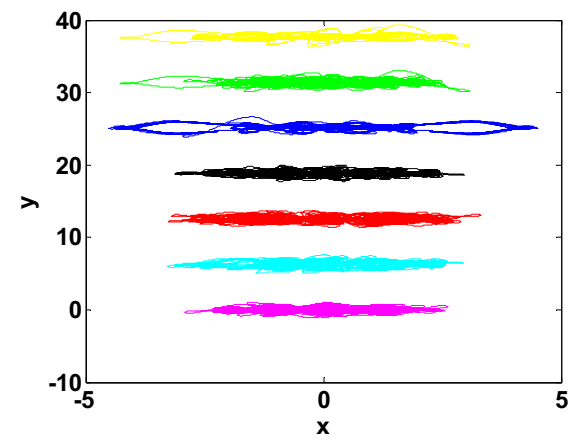

Figure 5. Coexistence of attractors under initial conditions $(2, \pm 2 \mathrm{k} \pi, 5,1)$.

\section{Circuit implementation}
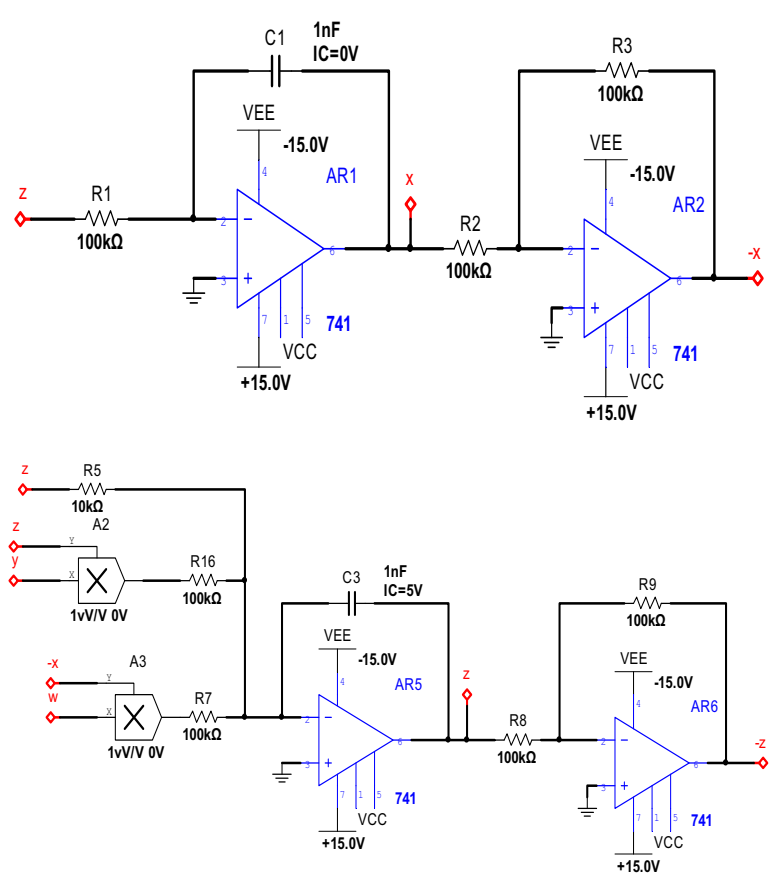

In this section, the experimental circuit of system (3) is designed, and the verification is completed through Multisim. The four-channel circuit is shown in figure 6 .
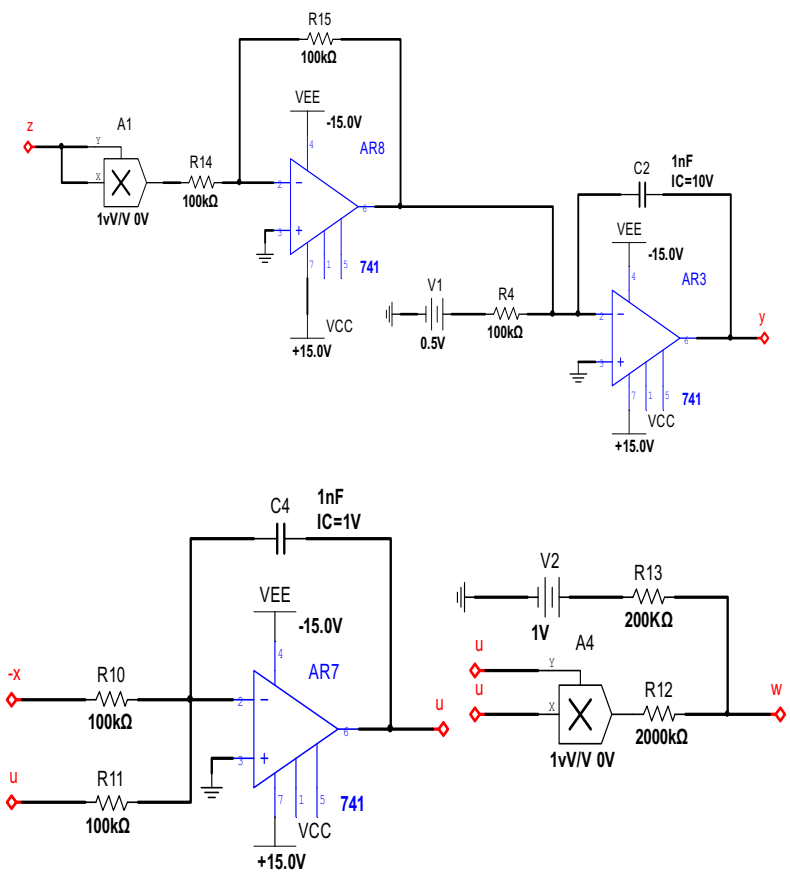

Figure 6. The circuit schematic of system (3)

The integrated circuit is designed by using general simulation calculation method. According to Kirchhoff's circuit law and the electrical characteristics of circuit elements, the multiplier is set as $1 / \mathrm{V}$, and the circuit in figure 7 can be represented by the equations (5) as follows:

$$
\left\{\begin{array}{l}
c_{x} \frac{d_{x}}{d_{t}}=-\frac{v_{z}}{R_{1}} \\
c_{y} \frac{d_{y}}{d_{t}}=\frac{v_{z} v_{z}}{R_{14}}-\frac{0.5 \mathrm{~V}}{R_{4}} \\
c_{z} \frac{d_{z}}{d_{t}}=\frac{v_{x}}{R_{7}}\left(\frac{1}{R_{13}}+\frac{v_{\varphi} v_{\varphi}}{R_{12}}\right)-\frac{v_{y} v_{z}}{R_{16}}-\frac{v_{z}}{R_{5}} \\
c_{\varphi} \frac{d_{\varphi}}{d_{t}}=\frac{v_{x}}{R_{10}}-\frac{v_{\varphi}}{R_{11}}
\end{array}\right.
$$

Where $v_{x}, v_{y}, v_{z}, v_{\varphi}$ respectively indicate the voltage of $\mathrm{x}, \mathrm{y}, \mathrm{z}$ and $\varphi$ on capacitor. Let $\mathrm{t}=\tau \mathrm{RC}$, where $\tau$ is the dimensionless time. The parameters are taken as follows: $\mathrm{C} x=\mathrm{C} y=\mathrm{C} z=\mathrm{C} \varphi=C, \mathrm{R}_{2}=\mathrm{R}_{3}=\mathrm{R}_{4}=\mathrm{R}_{7}=\mathrm{R}_{8}$ $=\mathrm{R}_{9}=\mathrm{R}_{10}=\mathrm{R}_{11}=\mathrm{R}_{14}=\mathrm{R}_{15}=\mathrm{R}, \mathrm{R}_{1}=\mathrm{R} / \mathrm{b}, \mathrm{R}_{5}=\mathrm{R} / \mathrm{d}, \mathrm{R}_{12}$ $=\mathrm{R} / \mathrm{m}, \mathrm{R}_{12}=\mathrm{R} / \mathrm{n}$, figure 7 shows the oscilloscope traces from the circuit. 

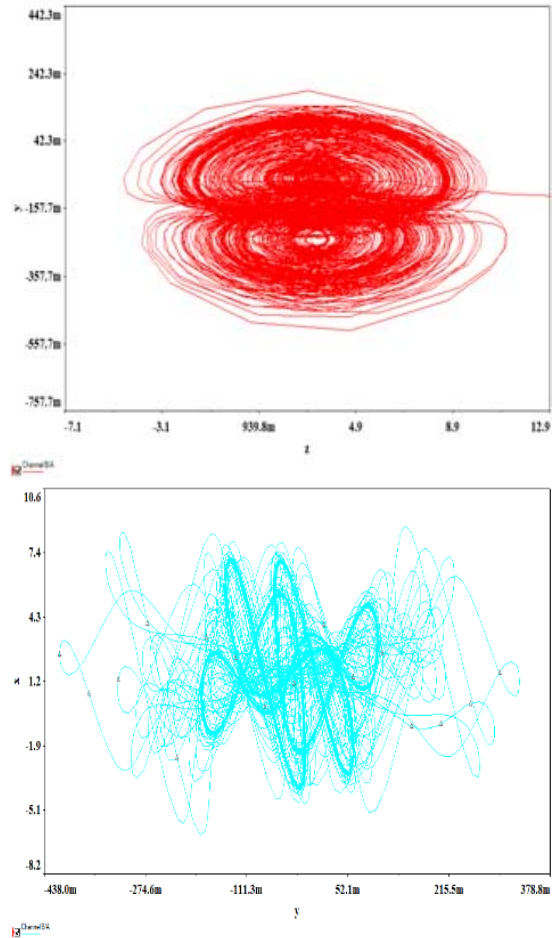

Figure 7. Phase trajectories of system (2) shown in Oscilloscope.

\section{Conclusions and discussion}

In this paper, we studied the basic dynamic characteristics of a nonequilibrium memristor system, including phase diagram, Lyapunov exponents, bifurcation diagram, etc., and transform the system so that it can realize the polarity control from two levels. In order to verify the feasibility of the system, the equivalent circuit of the memristor system is designed, and the experimental results are consistent with the numerical simulation and theoretical analysis results.

\section{Acknowledgments}

This work is supported by the Natural Science Foundation of Shandong Province (Grant No. ZR2017PA008), Shandong Province Key Research and Development Plan (2019GGX104092).

\section{References}

1. Strukov D B,Snider G S,Stewart D R,Williams R S. (2008) The missing memristor found. J. Nature, 453:80-83.

2. Zhou L,Wang C.H, Zhou L.L. (2018)Generating Four-Wing Hyperchaotic Attractor and TwoWing, Three-Wing, and Four-Wing Chaotic Attractors in 4D Memristive System. J, Int.J.Circ.Theor,46:84-98.

3. Wang, N. , Zhang, G.S, \& Bao, H. (2019). Bursting oscillations and coexisting attractors in a
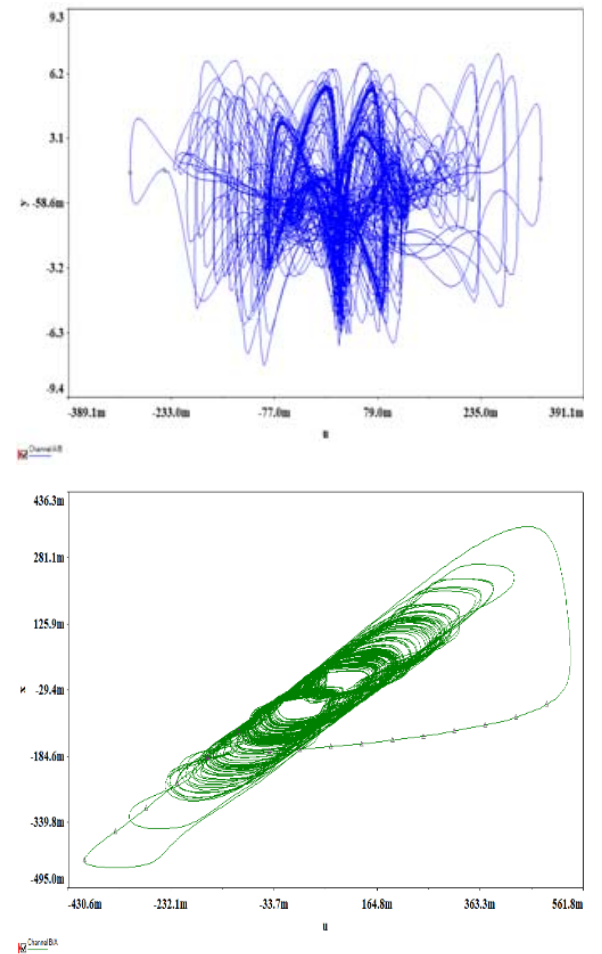

simple memristor-capacitor-based chaotic circuit. J. Nonlinear Dynamics, 97(2):1477-1494..

4. Li C, Sprott J C, Xing H. (2017).Constructing chaotic systems with conditional symmetry. J. Nonlinear Dynamics, 87(2):1351-1358.

5. Galias Z. (2018) .Study of amplitude control and dynamical behaviorsof a memristive band pass filter circuit.J. IEEE Trans Circuits Syst II, 65(5):637-641.

6. Bayani A, Rajagopal K, Khalaf A J M, et al. (2019).Dynamical analysis of a new multistable chaotic system with hidden attractor: Antimonotonicity, coexisting multiple attractors, and offset boosting[J]. Physics Letters A, 383(13):1450-1456.

7. Sun C.C, Chen Z.T, Hou X.L. (2017). Dynamical analysis and vibration control of a novel chaotic system with infinite equilibria. J. Journal of vibration and shock, 36(21):220-224+231.

8. Dang X Y,Li C B, Bao B C. (2015).Complex transient dynamics of hidden attractors in a simple 4D system. J. Chinese Physics B, 24(05):274-278.

9. Borah, M., \& Roy, B. K. (2017). Hidden attractor dynamics of a novel non-equilibrium fractionalorder chaotic system and its synchronisation control.In: Indian Control Conference (ICC) .Indian. 450-455.

10. Li, C. , Thio, J. C., Sprott, J. C. , Iu, H. H. C. , \& $\mathrm{Xu}, \mathrm{Y}$. . (2018). Constructing infinitely many attractors in a programmable chaotic circuit.J. IEEE Access, 1-1. 